Revue

Revue de l'histoire des religions

de Ihistoire des religions

1 | 2016

Varia

\title{
Les œuvres et la foi : une querelle poétique autour de la prédestination dans le Maroc du XIX ${ }^{\mathrm{e}}$ siècle
}

Works and Faith: A Poetical Dispute over Predestination in $19^{\text {th }}-C$ Morocco

\section{Youcef Djedi}

\section{OpenEdition}

Journals

Édition électronique

URL : http://journals.openedition.org/rhr/8484

DOI : $10.4000 /$ rhr.8484

ISSN : 2105-2573

Éditeur

Armand Colin

Édition imprimée

Date de publication : 1 mars 2016

Pagination : 49-69

ISBN : 978-2-200-93059-2

ISSN : 0035-1423

\section{Référence électronique}

Youcef Djedi, « Les œuvres et la foi : une querelle poétique autour de la prédestination dans le Maroc du xix ${ }^{e}$ siècle », Revue de l'histoire des religions [En ligne], 1 | 2016, mis en ligne le 01 mars 2019,

consulté le 05 septembre 2020. URL : http://journals.openedition.org/rhr/8484 ; DOI : https://doi.org/ $10.4000 /$ rhr.8484 


\section{Les œuvres et la foi : une querelle poétique autour de la prédestination dans le Maroc du XIX ${ }^{\mathrm{e}}$ siècle}

La doctrine du qadar est le plus souvent examinée sous sa forme de prédétermination. Elle est moins connue sous sa forme de double décret, en vertu duquel Allah a de toute éternité décidé la "félicité» de quelques-uns et la « réprobation" du reste du genre humain. Ce problème, indissociable de celui de la "foi» et des «œuvres», resurgissait assez régulièrement dans l'histoire islamique, et peut-être plus souvent encore au Maghreb, en raison de son passé almohade. Se pose aussi la question de la certitude du salut - ou de la réprobation -, ce dont nous fait également écho la poésie «populaire», ou melhoun.

\section{Works and Faith:}

\section{A Poetical Dispute over Predestination in $19^{\text {th }}-\mathrm{C}$ Morocco}

The doctrine of the qadar is most often examined in the form of predetermination, for it is less well known as the everlasting double decree through which Allah decided upon the "bliss" of some men and the "condemnation" of the rest of mankind, for all eternity. This problem, inseparable from that of "faith" and "works", reappeared rather regularly throughout Islamic history, and perhaps more often still in the Maghreb because of its Almohad past. Another matter arose also, that of the certainty of salvation - or damnation -, as echoed in "popular" poetry, or melhoun. 


\section{INTRODUCTION}

Il y a dans la littérature dite improprement «populaire » du Maghreb un fonds très peu exploité par les sciences sociales et humaines. Improprement disons-nous, parce que le melhoun - c'est en effet le nom le plus communément admis - n'a de populaire que la structure dialectale de sa langue par rapport à une langue arabe académique productrice d' $a d a b$ et de «sciences religieuses». Pourtant le registre du melhoun aussi est incontestablement savant, et de bien grands esprits, qu'ils fussent artisans, hommes de plume ou de sabre, s'y étaient exercés, voire des princes et des sultans. Certains d'entre ces poètes avaient même bravé parfois le paradoxe, pour ainsi dire, en faisant du melhoun en arabe littéral. Ainsi Saïd ben Abdallah el-Mendassi (XVII $\left.{ }^{\mathrm{e}} \mathrm{s}\right)^{1}$, par exemple, ou Mohammed Benmsayeb $\left(\mathrm{XVII}^{\mathrm{e}} / \mathrm{XVIII}^{\mathrm{e}} \mathrm{s} .\right)^{2}$ ou même Abdelaziz el-Meghrawi (XVI $/ \mathrm{XVII}^{\mathrm{e}} \mathrm{s}$. $)^{3}$. Mais c'était surtout le cas du grand poète et chevalier de l'Ouest algérien, le saint de Mzila, Lakhdar Benkhlouf (XVII ${ }^{\mathrm{e}}$ s. $)^{4}$. C'est dire donc toute l'importance que recèle l'étude de cette littérature. Dans le peu de documents qui soient sortis des bibliothèques privées, le sociologue, autant que le juriste, le psychologue et le psychanalyste autant que le linguiste et le théologien y trouveraient bien des fois un matériau que la littérature «savante» a souvent cessé de fournir. L'historien politique ne sera certainement pas en reste et trouvera quelques thèmes, comme celui de l'amour mystique, ayant valeur sotériologique, que l'on cultive, principalement au Maroc, pour la «sainte famille», dont se réclament les dynasties successives, quoique sunnites et dans un environnement quasi exclusivement sunnite. Bref, «seule la mise au jour des horizons perdus, des langages oubliés de la durée maghrébine, peut permettre (...) l'intelligence de cette dernière et partant, sa modernisation véritable 5 .» Mais on n'étonnera personne en disant que l'amour

1. Ahmed Amine Dellai, Guide bibliographique du Melhoun, Paris, L'Harmattan, 1996, p. 176-181.

2. Ibid., p. 129-141.

3. Ibid., p. 241-244.

4. Ibid., p. 123-128.

5. Berque (J.), L’intérieur du Maghreb, Paris, Gallimard, 1978, p. 539. 
théosophique et l'amour érotique tiennent la première place dans la poésie populaire, qui, précisément, s'avère une source incomparable en la matière. Quiconque, en effet, connaît un tant soit peu cette poésie peut voir sans trop de hardiesse la richesse et la profondeur qui la caractérisent, mais aussi la vie qui l'anime en comparaison avec la poésie savante devenue depuis longtemps, et jusqu'à l'époque qui nous occupe, et au-delà encore, souvent une simple versification sclérosée. Les pièces de Mohammed et de Boumédiène Bensehla $\left(\mathrm{XVIII}^{\mathrm{e}} \text { s. }\right)^{6}$, Mohammed Benmsayeb, Ahmed Bentriki $\left(\mathrm{XVII}^{\mathrm{e}}{ }^{\mathrm{s} .}\right)^{7}$, Abdelaziz el-Meghraoui, Mohammed el-Mesmoudi $\left(\mathrm{XVII}^{\mathrm{e}} \mathrm{s} \text {. }\right)^{8}$ et de tant d'autres, sont une mine d'informations sur les mœurs de l'époque, sur les rapports entre les genres et l'érotisme, mais aussi sur la fluidité des frontières entre l'amour des femmes, l'amour du Prophète, l'amour des saints, voire de la Kaaba et, enfin, l'amour de Dieu, auquel, avec l'amour du Prophète, Lekhal Lekhdar Benkhlouf, avait donné ses lettres de noblesse. Et pour tout dire, la poésie populaire a carrément pris le relais sur des sujets comme l'amour courtois et, surtout, le fameux «juge des amoureux» [qadi nas el-ghram], devant lequel on «traîne» son «bien-aimé», que celui-ci soit du reste une femme ou un saint, comme le fait Abdelaziz el-Maghraoui, par exemple, avec Abdelkader el-Gilani, pour le rappeler à la très épineuse «équivalence» affective ${ }^{9}$.

\section{$S_{A}{ }^{6} \bar{A} D A$ ET $C H A Q \bar{A}{ }^{\prime}$ OU LE DOUBLE DÉCRET DE LA PRÉDESTINATION : L'HÉRITAGE ALMOHADE}

Le point qui nous intéresse ici est d'un tout autre genre et ne manque pas du tout de piquant. La querelle sur laquelle il porte est de celles qui ont toujours secoué l'islam, depuis ses débuts, quoiqu' apparemmentbeaucoupplus attachéeàl'histoiremaghrébine, en raison de l'épisode almohade dont elle reste fortement marquée. Certes, ce dogme est, dans son principe même, incontournable dans

6. A. A. Dellai, op.cit ., p. 145-147.

7. Ibid., p. 151-155.

8. Ibid., p. A. A. Dellai, op.cit ., p. 244.

9. "قاضي الهوى باحكامه حكم * كما رضيناك ارضاني. Sur le problème de l' «équivalence» en théologie musulmane, cf. notamment Ignaz Goldziher, «Die Gottesliebe in der islamischen Theologie», in Der Islam (1919), IX, p. 144 sq. 
la religion islamique, et personne n'oserait le nier sans encourir l'opprobre et la vilénie, comme cela a été le cas des qadarites et des mu $u^{<}$tazilites. Seulement, les tendances «atholiques $»^{10}$ qui, au fil des siècles, renforçaient l'orthodoxie doctrinale, avaient neutralisé, en quelque sorte, ce decretum horribile, au point que le salut le plus universellement admis dans l'islam s'obtient désormais d'abord par la foi.

Au regard de cette réalité, l'épisode almohade apparait, non seulement comme un contraste politique, mais aussi bien théologique. Pour ce qui nous concerne, la quasi-totalité des documents connus relatifs à l'almohadisme sont, en effet, empreints de références manifestes à la double prédestination. On peut s'étonner, à ce propos, que l'on n'ait pas encore très bien mis au clair, ni que l'on ait même mis le doigt sur la place de cette doctrine dans l'idéologie mouminide, c'est-à-dire l'idéologie sur laquelle s'était bâtie la dynastie fondée par Abd al-Moumène ibn Ali el-koumi (1094-1163), bras armé et successeur de Mohammed ibn Toumert, véritable initiateur de l'extraordinaire épisode almohade (1121-1276).

De toute évidence, on s'était nourri alors aussi bien d'enseignements directs et de pratiques de ce dernier que de son livre, qui contient une nette profession de foi prédestinatianiste, puisque «tout ce qui vient à l'existence $[. .$.$] dans le royaume de Dieu est déjà le fait de sa$ prédestination, sabaqa bihi qad $\bar{a}^{>} u h$ wa qadaruh. [...] Rien ne saurait être retardé, ni avancé, et [...] à celui qui a été créé pour la félicité, $n a^{<} \bar{\imath} m$, il sera aisé de faire le bien, yuyassar li-l-yusrā, tandis qu'à celui qui a été créé pour l'enfer, ğahīm, il sera facilité de faire le mal, yuyassar $l i-l-<u s r a \bar{a}$. On est bienheureux [sa<id] ou réprouvé [chaqiyy] déjà dans le ventre de sa mère. Tout cela par la prédestination de Dieu $^{11}$.» Il va sans dire que toute la terminologie utilisée ici par l'Imam est la même que l'on retrouve dans les principaux recueils sunnites de hadiths, Būkhāri et Muslim à leur tête.

Il est vrai aussi que cette déclaration semble comme noyée dans ce livre; c'est ce qui explique peut-être que, dans l'«exposé

10. Goldziher (I.), « Katholische Tendenz und Partikularismus im Islam », in Beiträge zur Religionswissenschaft (1913/14), I, nº 2, p. 115-142.

11. $A^{<} a z z, m \bar{a}$ yutlab, Alger, Carbona, 1903, p. 236. Peu nous importe ici que la copie parvenue à nous soit l'original authentique ou qu'elle ait subi des rajouts. Cf. Maribel Fierro, «Le mahdi Ibn Tûmart et al-Andalus : l'élaboration de la légitimité almohade», Revue des mondes musulmans et de la Méditerranée, p. 107-124. 
désordonné et touffu» (Mohamed Talbi), qui servit d'introduction à ce livre, Goldziher se soit fourvoyé en montrant les rapports du Mahdī des Almohades avec Ghazzālī et se soit contenté d'une vague allusion au «petit groupe de Maghrébins élus que Dieu a appelés à la conquête du monde $»^{12}$. Mais à la décharge du savant hongrois, on peut en dire autant d'Évariste Lévi-Provençal, qui avait complètement ignoré la place cruciale de la doctrine du double décret chez ibn Toumert et ses successeurs, alors que, spécialiste de l'époque, il était l'éditeur de nombreux documents almohades au discours très franchement prédestinatianiste. Des auteurs plus récents encore n'ont pas été plus attentifs à cette particularité doctrinale et politique de l'almohadisme.

De fait, même ce qui est admis comme ayant été une révocation de la doctrine d'ibn Toumert par le calife almohade al-Mamoune en 1230 ne concernait en fin de compte que le statut d'imam infaillible de ce dernier et son caractère messianique. Et lorsqu'on voit les différents documents officiels postérieurs ${ }^{13}$, ou même une simple relation de voyage d'un fonctionnaire almohade comme ibn Djubayr ${ }^{14}$, dont les références à la double prédestination de Dieu sont aussi nombreuses que précises, c'est bien tout le contraire d'une influence de Ghazzālī que l'on pourrait conjecturer sur ce point précisément: le grand Docteur aurait, en effet, très certainement dénoncé l'utilisation de la doctrine de la prédestination faite par les Almohades, depuis ibn Toumert lui-même, dans leur propagande, mais surtout dans le recrutement et l'encadrement de leurs partisans, comme il l'avait fait précisément dans son pamphlet dirigé contre les Bāținiyya ${ }^{15}$.

Car il faut le dire, cette doctrine a bien été le levain révolutionnaire de ces puritains maghrébins, comme elle sera, quelques siècles plus tard, celui des calvinistes en Europe du Nord. L'invincibilité des cavaliers de Cromwell est connue, comme sont connues aussi leurs convictions

12. Le livre de Mohammed Ibn Toumert Mahdi des Almohades, Alger, P. Fontana, 1903, p. 100. Goldziher avait déjà consacré deux articles à l'almohadisme: «Materialen zur Kenntnis der Almohadenbewegung in Nordafrika», in Zeitschrift der Deutschen Morgenländischen Gesellschaft [Z.D.M.G]. (1887), XLI, p. 30-140 et «Die Bekenntnisformeln der Almohaden», in Z.D.M.G. (1891), XLV, p. 168-171.

13. Inter alia Lévi-Provençal (É.), «Six fragments inédits d'une chronique anonyme du début des Almohades», in Mélanges René Basset, t. II, Paris, E. Leroux, 1925, p. 335-393; Id., Trente-sept lettres officielles almohades, Rabat, Institut des Hautes Études Marocaines, 1941.

14. Rihla, Alger, Mūfam li-n-našr, 1988, passim.

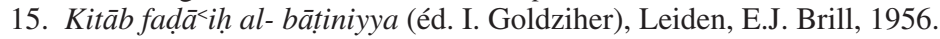


prédestinatinaistes. De même, les combattants mouminides étaient craints jusqu'au Moyen-Orient et au-delà, parce que l'une de leurs motivations, et non des moindres, est d'être le «parti de Dieu» et ses «élus», qu'il a, de toute éternité, prédestinés à bien. Une telle croyance, qui a fait ses preuves, ne pouvait certainement pas laisser indifférentes les dynasties post-almohades; toutes ont recouru, peu ou prou, à cette même méthode de légitimation et d'embrigadement qui avait fait le succès fulgurant de leurs devanciers.

Quoi qu'il en soit, la doctrine du double décret de la prédestination est quasiment oubliée à l'époque moderne, aussi bien dans le protestantisme que dans l'islam. Il n'est pas jusqu'aux termini technici mêmes, sa' $\bar{a} d a$ et chaqā', qui ne soient devenus inconnus dans l'islam moderne. Celui-ci voit bien plutôt la consécration, par la force des choses, d'une victoire tardive du principe mu $\mathbf{r}^{<}$tazilite du libre arbitre, que l'on admet de plus en plus, comme pour conjurer la menace du fatalisme ${ }^{16}$. Il n'empêche, pourtant, que le dogme de la double prédestination est inséparable des dogmes fondamentaux de la foi islamique, comme le montrent les événements que nous allons présenter.

\section{LA FOI SANS LES GEUVRES?}

« Notre Seigneur a créé le bienheureux bienheureux et le réprouvé réprouvél $\mathrm{Ne}$ te soucie (donc) pas de (tes) péchés et ne compte pas sur une quelconque piété/ Notre Seigneur est riche et compatissant. $\gg^{17}$

16. Cf. e.g. la conférence de La Mecque (1899) consacrée notamment au problème du fatalisme (Friedrich Ulrich, Die Vorherbestimmungslehre im Islam und Christentum, Université de Heidelberg, 1912, p. 10, 13, 130-131). Cf. également Jacques Jomier, Le commentaire coranique du Manâr. Tendances modernes de l'exégèse coranique en Égypte, Paris, Maisonneuve \& Cie, 1954, p. 137-138; Carl Heinrich Becker, «Islam », in Archiv für Religionswissenschaft (1912), XV, p. 536-537; Roger Arnaldez, in Encyclopaedia Universalis [E.U.], s. v. «Mu'tazilisme», et surtout l'anecdote de Henrik Samuel Nyberg et la remarque de Hellmut Ritter, dans Classicisme et déclin culturel dans l'histoire de l'Islam, Paris, Maisonneuve \& Larose, 1977, p. 182.

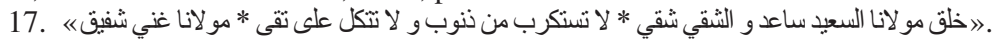
Comparer avec la traduction proposée par Mohammed al-Fasi in Cahiers du Sud (1940). 
Le grand poète de Meknès, Abdelkader (plus couramment Kaddour) el-Alami (1742-1850) $)^{18}$ fait écho ici à une grande controverse, survenue dans la première moitié du dix-neuvième siècle, autour de la question de la foi et de l'efficace des œuvres. À l'origine, l'affaire, qui en apparence ne portait pas directement sur la prédestination, fut déclenchée par la polémique entre deux de ses jeunes contemporains et grands poètes, el-Madani Tourkmani (1815-1885) ${ }^{19}$ de Marrakech et Ahmed el-Ghrabli ${ }^{20}$ de Fès. Mais en vérité c'était bien le problème de la prédestination qui resurgissait une fois encore.

Voici le fond de l'affaire: Ghrabli, relayant certainement quelque débat houleux sur la question, avait déclaré qu' «on ne peut se suffire du témoignage (de la foi) sans les œuvres ${ }^{21} »$. Tourkmani appela dès lors son «contradicteur à témoigner (de sa foi), car le témoignage de (l'unicité) de Dieu et de (la véracité) de (son) apôtre suffit, a suffi et suffira; c'est un bien ici-bas et davantage encore dans l'au-delà * Et l'intention (sincère) du fidèle vaut mieux que ses œuvres ${ }^{22}$.» Certes, réplique le poète de Fès,

«l'intention (sincère) est à l'origine des œuvres, et celui qui demande une chose elle lui sera donnée * Celui qui ignore sa Loi dans ses moindres significations est perdu * [...] décris donc les fondements de la foi, ô toi qui as perdu son capital * Et décris les œuvres si tu les as étudiées * [...] Promène-toi et demande si celui qui ne donne plus d'impôt légal, ni ne purifie ses biens * A vécu dans le péché, ou est-ce que le sens de sa profession de foi lui aura suffi * Promène-toi et demande si celui qui n'observe plus le jeûne et les conditions de sa perfection sera châtié, ou est-ce que le sens de sa profession de foi lui aura suffi ${ }^{23}$.»

Et ainsi du pèlerinage et du reste des observations cultuelles. Mais cette obstination à ignorer l'efficace de la foi fait monter la

18. Ahmed Amine Dellai, op.cit., p. 202 sq.

19. Ibid., p. 258-259.

20. Ibid., p. 235-236.

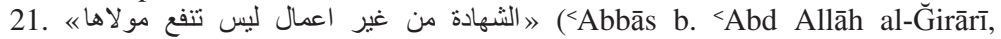
al-Qașìda, Rabat, Maktbat at-țālib, 1970, p. 412).

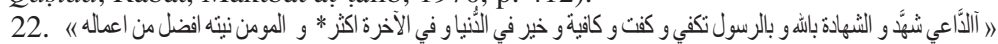
(ibid., p. 412).

23.

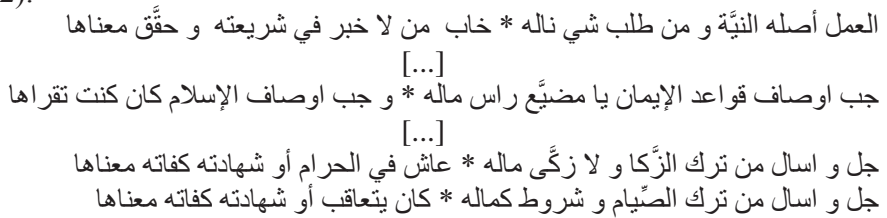

(Ibid., p. 413-415) 
tension d'un cran. C'est d'ailleurs le propre des querelles sur la prédestination que d'en arriver parfois même aux mains, comme autrefois entre les Wașilites et les Ibāḍites sous le règne des Rustumides en Algérie ${ }^{24}$, ou à l'époque de la Réforme, à Zurich, entre Peter Martyr Vermigli et Theodor Bibliander ${ }^{25}$. Peut-être était-ce l'éloignement qui avait évité aux deux poètes marocains des solutions aussi extrêmes. Car, sinon pour ce qui était des avanies, elles allèrent bon train. Après avoir été traité d'impie et d'ignorant aveuglé par $\operatorname{Satan}^{26}$, Tourkmani riposte de plus belle en traitant à son tour son antagoniste de «niais», de «dément» et même d' «âne, fils d'âne, portant sur son dos un (morceau de) bois brut $»^{27}$ - Calvin traitait ses adversaires et ceux de la prédestination de «chiens enragez qui desgorgent leurs ordures à l'encontre de la majesté de Dieu ${ }^{28}$.» Est-ce là une allusion de la part de Tourkmani à «l'âne portant des livres» du Coran ${ }^{29}$ ? Peut-être. Toujours est-il qu'il finit par presser son adversaire de se rendre à ses arguments:

«Demande pardon à Dieu, si tu es un authentique musulman * Ne mets rien au-dessus de la profession (de foi) * Car elle est au-dessus de tout * Pas même l'action la plus parfaite ne saurait la surpasser * Les œuvres et la foi ne valent qu'ensemble * A quoi ont servi les œuvres à plus d'un aveuglé * Qui a œuvré jusqu'à apercevoir la réussite * Et qui, (après) avoir suivi ses penchants, est mort impie par ses (propres) actions ? Et ainsi, à l'inverse, plus d'un a goûté, après la mécréance, le miel de la douceur de la foi en toute connaissance $*$ Et le jour où il a été guéri de l'impiété * Il a fait profession de (l'unicité de) Dieu et de (la véracité de ce qui a été) transmis par le Prophète * On a dit qu'il est mort en martyr, grâce à elle, et quiconque la prononce dans sa vie s'en ira à coup sûr au paradis ${ }^{30}$.»

24. Gérard Dangel, L'imamat ibadite de Tahert (761-909). Thèse, Université de Strasbourg, 1977, p. 88-89, 140-141 ; I. Goldziher, «Materialen...», op.cit., p. 34-35.

25. Joachim Staedke, «Der Zürcher Prädestinationsstreit von $1560 »$, in Zwingliana (1949/53), IX, p. 544-555.

26. <A. al-Ğirārī, op.cit., p. 413-415.

27. Ibid., p. 416.

28. Ap. Lucien Febvre, Le problème de l'incroyance au $16^{e}$ siècle, Paris, 1942, p. 440 n. 116.

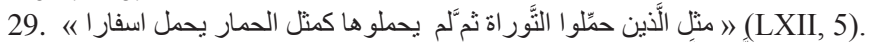

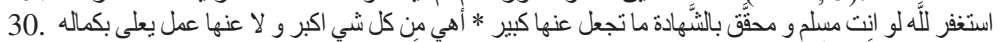

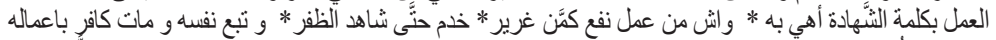

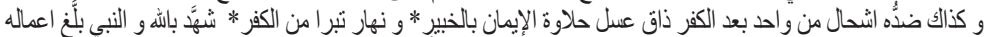

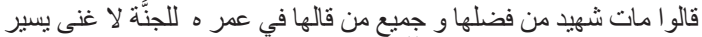
(‘A. al-Ğirārī, op.cit., pp. 416). 
Tourkmani, à l'évidence, tire la force de ses arguments du même hadith - et de ses variantes - qui enseigne également, en effet, que quiconque est créé pour le paradis retrouvera inéluctablement les œuvres des élus, quand bien même il aurait passé toute sa vie dans le péché et qu'il ne lui resterait que la distance d'un bras le séparant de l'enfer; et inversement, celui prédestiné à la damnation éternelle finira immanquablement par accomplir les œuvres des réprouvés, dût-il avoir passé une vie impeccable et qu'il ne lui resterait que la distance d'un bras le séparant du paradis. La seule certitude qui doive être retenue est donc celle sur laquelle on meurt, la note finale, pour ainsi parler, la khätima ${ }^{31}$. Sous cet angle, on pourrait dire que Victor Hugo, par exemple, avait fait preuve d'une intuition remarquable de l'éthique musulmane, en faisant mourir son «Sultan Mourad»sur une œuvre pie: toute sa vie de monarque impitoyable et incrédule avait été effacée par ce geste du pied par lequel il avait chassé les mouches de devant un porc mourant. Mais on pourrait voir aussi dans cette khātima, non pas l'accomplissement incontournable de la prédestination, mais un acte in extremis de la miséricorde divine ${ }^{32}$.

Tout comme dans l'Europe protestante, le débat réapparaissait régulièrement dans l'aire culturelle islamique, et peut-être de façon plus marquée dans sa partie occidentale en raison de son passé almohade. Au XVII ${ }^{\mathrm{e}}$ siècle, par exemple, l'Oriental Ibrahim ibn Hassan Chahrazouri avait dû essuyer, de la part des théologiens de Fès, «la plus acerbe critique et fut accusé d'avoir commis une faute impardonnable» en professant des opinions peu orthodoxes sur le libre arbitre ${ }^{33}$. C'est qu'il en coûtait, au Maghreb surtout, d'emprunter les voies du mu<tazilisme ${ }^{34}$, lequel n'était pas seulement synonyme

31. Louis Gardet, «Les noms et les statuts. Le problème de la foi et des œuvres en Islām», in Studia Islamica (1956), V, p. 105-106.

32. Comme le laissent à peu près entendre les paroles de pénitence du héros de Harīīī, par exemple, dont la fin approche. En effet, tout en confessant la vérité de l'immarcescible prédestination, le mourant n'en espère pas moins un acte de grâce, un tawfíq, du Ciel qui sera pour lui comme un signe de rédemption avant de rendre l'âme (Maqāmāt al-Harīiñ, Beyrouth, Dar Șādir, 1980, p. 456 et n. 6).

33. Mohamed Ben Cheneb, «Biographies des principaux personnages mentionnés dans l'idjazah du cheikh ‘Abd al-Qâdir al-Fâsy», in Actes du XIV ${ }^{e}$ Congrès International des Orientalistes (1905), III, § 7; Jacques Berque, «La littérature marocaine et l'Orient au XVII' siècle», in Arabica (1955), II, p. 303.

34. Cf. e.g. <Abd ar-Razzāq ibn Hamādūš, Riḥla, Alger, Iṣdārāt al-maktaba al-wațaniyya, 1983, p. 66-67. 
de rébellion et de sédition, comme l'avait remarqué Goldziher ${ }^{35}$, mais pouvait aller jusqu'à avoir toute la charge de l'insulte et de l'infamie ${ }^{36}$.

Tourkmani, qui était partisan de la sola fides et de la prédestination gratuite, niait que l'on pût obtenir le salut par les œuvres. Il tenait pour efficace et «suffisante l'attestation [chahāda] (de l'unicité) d'Allah et (de l'authenticité de la mission) de l'Apôtre. ${ }^{37}$ Et si c'est la foi seule qui sauve, seuls comptaient donc à ses yeux ce à quoi l'on est prédestiné [sābiqa ${ }^{38}$ et ce sur quoi on devrait mourir, c'està-dire la khātima ${ }^{39}$. Ghrabli, au contraire, et bien que l'orthodoxie vît depuis toujours dans la foi l'unique attribut salvifique, tenait pour impie quiconque oserait «libérer»l'homme des œuvres, le ‘amal, parce que la profession de foi sans les œuvres est tout simplement insuffisante pour s'assurer le salut, car pour faire une épée, «quel sens aurait la poignée [à elle seule], sans la lame et aiguisée ? $^{40}{ }$ 》 Ghrabli, ce faisant, pourrait aisément se réclamer de l'ibāḍisme ou même du calvinisme. On sait bien, pour rester dans le giron de l'islam, à quel point l'ibāḍisme, rejeton du premier schisme dans l'islam, le kharidjisme, et qui subsiste jusqu'à nos jours en Afrique du Nord et à Oman, abhorre toute séparation entre foi et œuvres ${ }^{41}$.

Mais les œuvres défendues par le poète de Fès étaient celles que défendait Pighius contre Calvin. La question du statut des

35. Ap. R. Arnaldez, loc.cit.

36. Cf. Hady Roger Idris, «Essai sur la diffusion de l'aš‘arīsme en Ifrîqiya», in Cahiers de Tunisie (1953), $\mathrm{n}^{\circ}$ 1, p. 138 sur ce vieillard mort en non musulman parce que précisément mort mu<tazilite. La célèbre qașìda populaire «al-Ḥarrāz» (Ahmed Amine Dellai, Chansons de la Casbah, Ager, ENAG, 2000, p. 185) de Makkī [i]bn al-Quršī, mort en 1934, (notamment id., Guide..., op.cit., p. 323-324; <A. al-Girārī, op.cit., p. 126, 139, 686; Muhammad al-Fāsī, Ma lamat al-malhūn, Rabat, Maṭbū<āt akādīmiyyat al-mamlaka al-mag̉ribiyya, 1997, I, p. 146 ,42; II, p. 73 sq.; III, p. 323 sq.) nous a gardé une trace de la charge d'abjection contenue dans le mot «mu tazilī», qui y est synonyme d'impiété, parce que «tu n'as pas foi en une partie de l'islam» [k[a]fart(a) bi-ba ${ }^{<}$al al-[i]slām]. C'est presque en ces termes que le «fou» de la «Maqāma māristāniyya» s'adresse au narrateur accusé de mu<tazilisme (Abū-l-Faḍl Badī ${ }^{<}$az-Zamān al-Hamadhānī, Maqāmāt, Beyrouth, Imprimerie Catholique, 1956, p. 134).

37. <A. Malhūn̄î, op.cit., p. 81.

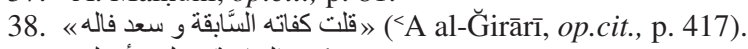

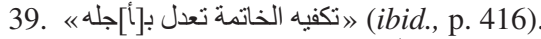

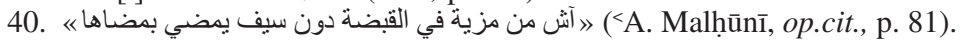

41. Cf. e.g. Djedi (Y.), compte rendu de Virginie Prevost, Les Ibadites. De Djerba à Oman, la troisième voie de l'Islam, Turnhout, Brepols, 2010, in Revue Européenne des Sciences Sociales (2012) 50-1, p. 281-283. 
œuvres est en tout cas assez vieille, et toujours elle draine avec elle les épineux problèmes de la prédestination, de la foi et de son efficace. Au fond, Tourkmani ne rejetait pas plus l'importance des bonnes auvres - il le dit ouvertement ${ }^{42}$ - que son contradicteur la vérité de la double prédestination. On n'oubliera pas non plus que la déferlante mystique y apporta aussi sa touche indélébile de prédestinatianisme. C'est ce qu'on voit aisément chez des poètes «populaires» nord-africains qui, pour avoir aussi toujours baigné dans le soufisme, affichaient constamment une croyance claire dans le double décret. Ils se disent prédestinés à l'amour de Dieu, du Prophète, de la «sainte famille», voire des lieux saints, dès avant l'âge canonique d'observer le jeûne obligatoire, c'est-à-dire avant la puberté, depuis la tendre enfance. Subtil euphémisme pour ne pas dire depuis «le ventre de la mère » comme le voudrait le célèbre hadith selon lequel : «le bienheureux est celui qui l'a été dès le ventre de sa mère, et le réprouvé est celui qui l'a été dès le ventre de sa mère » [as-sa`īd man sa ${ }^{<i d a ~ f i ̄ ~ b a t ̦ n ~ u m m i h ~ w a-c h-c h a q i y y ~ m a n ~}$ chaqiya fĩ baṭn ummih].

Ainsi, au XVI ${ }^{\mathrm{e}}$ siècle, dans l'une de ses nombreuses qasī $d a(\mathrm{~s})$ pleines de ferveur et d'amour pour le Prophète, Lakhdar Khlouf lance-t-il à l'adresse de son «sauveur» une déclaration d'amour mystique valant une vraie proclamation d'élection et de salut personnel: «Avant même (l'âge) de l'obligation du jeûne, Dieu m'infligea (ton amour) * Comme il avait infligé l'amour de la félicité future ${ }^{43}$ à Ḥassān [scil.

42. <A. Ğirārī, op.cit., p. 417.

43. Et non pas «de même qu'il consuma celui de Hassan (ben Tsabit) de son ardeur pour S'âda», selon la traduction de Joseph Desparmet («La poésie arabe actuelle à Blida et sa métrique », in Actes du Congrès International des Orientalistes (1905), $3^{\mathrm{e}}$ section, p. 595). La tradition est bien connue selon laquelle le jour du Jugement, le barde du Prophète, Hassān ibn Thābit, prendra la tête des poètes (pré) destinés au paradis, tandis que les autres poètes s'en iront croupir en enfer avec Imru`al-Qays. C'est en ce sens précisément que le poète Mohammed en-Nedjdjar (A.A. Dellai, op.cit., p. 251-253), par exemple, rend hommage à Hassān ibn Thābit dans sa «Aarfawiyya», à la fin de laquelle, d'ailleurs, il demande à Allāh de ne compter «aucun musulman parmi les réprouvés» [muslim la techqih]. Mais c'était là des détails qui n'étaient peut-être pas à la portée de Desparmet tant, pour lui, «le sentiment religieux et la mélancolie de l'idée de la mort sont profonds dans l'âme indigène.» (loc.cit, p. 582 n. 1.) Il est intéressant de rappeler qu'à l'époque de Benkhlouf les débats en Europe sur la prédestination étaient en train de participer à la construction de la civilisation moderne, comme l'avaient montré déjà les travaux précurseurs de Max Weber et de Ernst Troeltsch. 
ibn Thābit $]^{44}$.» Déclaration d'élection, disons-nous, parce que notre poète est on ne peut plus prédestinatianiste, Dieu ayant décidé de toute éternité, et une fois pour toutes, du sort final de tout un chacun, qui pour la félicité, qui pour l'opprobre, éternelles. Jouant sur la fin du hadith que nous venons de citer, suivant laquelle «les plumes sont levées et secs les feuillets » [rufi<at al-aqlām wa ğaffat a-ṣ-ṣuhufu, il professe aussi que «L'encre est sèche quant aux élus et réprouvés * Et dans l'éternité la plume écrit avec la permission du Créateur ${ }^{45}$.»

Même déclaration chez son contemporain et non moins célèbre Abdelaziz el-Meghraoui, dont les convictions prédestinatianistes, on le verra encore, ne sont pas moins ancrées; lui aussi se dit touché par l'amour du Prophète «avant l'âge du jeûne ${ }^{46}$ ». Cet amour salvateur avait «assailli» aussi le poète marocain Abdelkader Ksardjdidi, par exemple, «avant même (l'âge canonique) du jeûne ${ }^{47}$. Et c'était à cet amour précisément que Ghrabli avait succombé lui aussi. Dans l'un de ses poèmes, «al-Malika», un de ces panégyriques religieux en l'honneur de La Mecque et de la «sainte famille» du Prophète, dont on s'est fait une spécialité en Afrique du Nord, Ghrabli tire une singulière fierté, confinant, comme chez Benkhlouf, Ksardjdidi et el-Meghraoui, à la certitudo salutis. Lui aussi déclare avoir cédé à l' "amour» dès l'enfance, avant même d'avoir atteint l'âge du jeûne obligatoire ${ }^{48}$. Ghrabli ne niait donc pas la vérité de la prédestination. Il lui préférait seulement la surenchère des bonnes œuvres ${ }^{49}$.

Évidemment, si nécessaire, on dépasserait même les limites du «ventre maternel» évoqué dans le hadith ci-haut, pour la pure et stricte prédestination éternelle. C'est ce que fait, en effet, Abdelaziz el-Meghraoui dans «Essabi el-Ghessani». Quoiqu'encore impubère,

44. شبل وجوب الصَّوم بك ربِّي بلاني * كيف بلا حسَّان بمحبَّة السَّعادة ) (J. Desparmet, loc. cit., p. 593).

45. جف "المداد بالأشقيا و السعدا * و القلم في الأزل يكتب باذن الباري) (A.A. Dellai, Guide..., op.cit., p. 125).

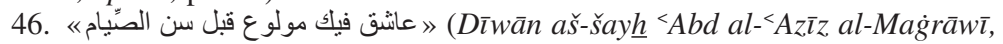
Rabat, Mațbū<āt akādīmiyyat al-mamlaka al-maġribiyya, 2008, p. 108. Cf. également ibid., p. 135).

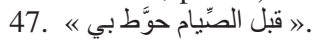

48. M. al-Fāsī, op.cit., I, p. 164.

49. <A. al-Ğirārī, op.cit., p. 412-415 (surtout p. 415 in fine). 
l'enfant en question se consume lui aussi d'amour pour le Prophète, un amour auquel il se dit prédestiné par un décret divin, consigné, depuis avant la création du monde, dans la «Table (bien gardée) $»^{50}$. Mais au fait pourquoi une telle généalogie de l'amour? Et d'abord, Dieu n'a-t-il pas déjà prédestiné toute la création? Sans doute, mais le fait même d'être une sorte de vase d'amour divin, décidé, qui plus est, par Dieu lui-même, peut être facilement déchiffré comme un signe indéfectible de la félicité future. Pour le reste, c'est l'expérience de la quête sotériologique qui - si elle n'est pas minée par le doute - confirmera durablement cette élection par l'amour mystique.

Est-ce à dire que l'amour profane soit le signe de quelque réprobation ? Non pas. Cette passion, qui est naturellement une totale prédétermination ${ }^{51}$, n'est pas pour autant un signe de prédestination à mal. Tout au plus pourrait-on dire qu'elle n'a pas de signification particulière pour les fins dernières, ainsi que le proclament distinctement ces vers de Ahmed Bentriki: "L'amour est une terrible affaire, à laquelle peut être prédéterminé, mqaddar, le réprouvé comme le bienheureux ${ }^{52}$.» Il en va d'ailleurs de même du «chrématisme» et du goût du lucre, par exemple, dont on fait actuellement grand cas avec la «théologie de la prospérité» de

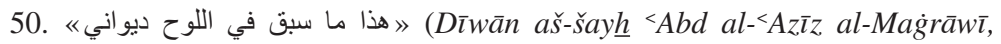
op.cit., p. 245).

51. Cf.e.g. Muhammad Bayțār, in Hamza Boubakeur, Trois poètes algériens,

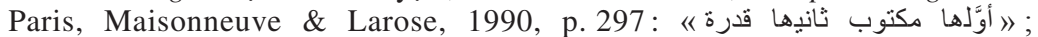
" و المكتوب اذا كتب يقضى محتوم (ibid., p. 299). Mais c'est là un principe commun de la poésie arabe, dans laquelle «inna-l-hawā qadaru», principe que l'on retrouve aussi à foison dans les poèmes chantés en musique andalouse: 《) (Chants andalous, Alger, ENAG/ Éditions, 2002,

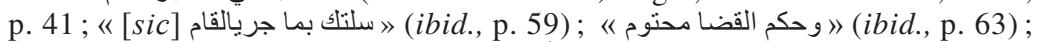

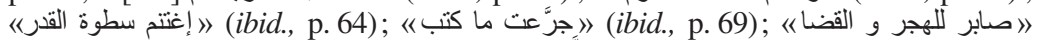

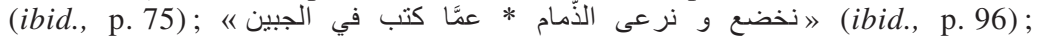

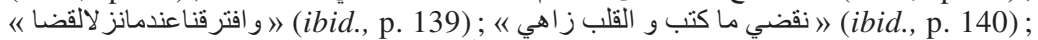

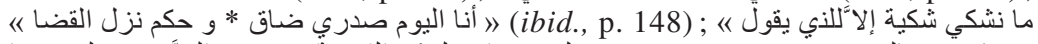

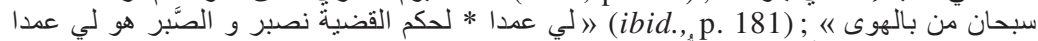

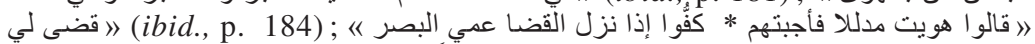

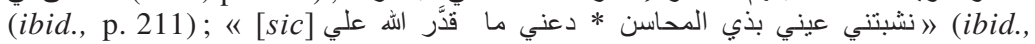
218). Et tout cela est bien évidemment dans la prédétermination universelle:

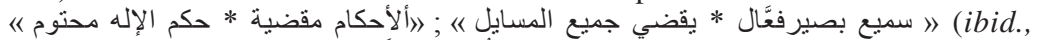

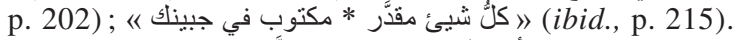

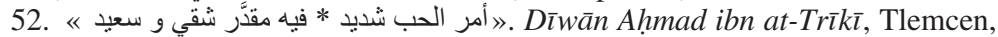
Édition Ibn Haldūn, s.d., p. 116. 
«l'islam de marché» ${ }^{53}$. C'est là encore Kaddour el-Alami qui tranche dans le vif dans sa qasīda, inédite, Essouk - ou Naqes lebdaa -, probablement, là aussi, en réponse à quelque controverse ayant pour objet la signification sotériologique de l'enrichissement et de la quête des biens terrestres: "Gloire à celui qui l'a créé et lui a dit: 'Soit, ô dinar, une tentation pour les êtres, (de telle sorte que) t'obéiront le réprouvé et le bienheureux ${ }^{\text {" }}{ }^{4}$.»

Ce qui est intéressant de savoir maintenant, c'est l'issue prise par la querelle entre Ghrabli et Tourkmani. Comme il fallait évidemment s'y attendre, on en appela à l'arbitrage d'une autorité «scientifique $»^{55}$. Ce fut à Mohammed Bouachrine, cadi de son état, que revint la charge de trancher dans le débat avec une «Incitation à l'observance du culte en réponse à celui qui fit preuve d'impiété à propos des deux professions de foi $»^{56}$. Mais d'abord, il est important de rappeler que l'intervention de Bouachrine avait été sollicitée par «certains notables [ba'd al-khawās] acquis aux propos de Ghrabli ${ }^{57}$, dont il semble au demeurant qu'on ne craignait pas uniquement la verve mordante, car le grand poète de Fès brillait aussi, dit-on, par de redoutables talents de sorcier ${ }^{58}$.

On devine déjà qui des deux contradicteurs devait faire les frais du verdict rendu par le «savantissime» magistrat. En un mot, Tourkmani, dit en substance l' «Incitation», n'est pas seulement un impudent [min al-mutahattikīn], séduisant par sa toile d'erreurs les esprits égarés, mais c'est aussi un sacrilège qui a fait preuve d'une «pure impiété», zandaqa mahd ${ }^{59}$. La réponse de Bouachrine s'était organisée autour du thème des œuvres et de la foi, c'est-à-dire celui

53. Cf. Patrick Haenni, L'islam de marché. L'autre révolution conservatrice, Paris, Seuil, 2005; Youcef Djedi, «Islam in Weber and Some Weberian Considerations on Islam and Modernity », in Sam Whimster et al., Max Weber and the Reconfiguration of Modernity, London (à paraître).

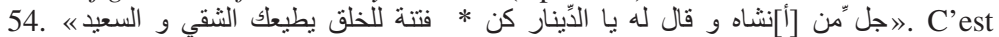
l'occasion pour moi de remercier ici très vivement M. Abdelghani Mehdaoui de Bordeaux, qui a mis à ma disposition cette pièce et de nombreuses autres du reste, qui ne sont pas encore éditées. Je le remercie tout autant pour ses remarques et ses suggestions.

55. <A. al-Malhūn̄̄, op.cit., p. 82.

56. ألحضُّلى العبادة في الرَّدّ على من ألحد في كلمني الثَّهادة 《. Ibid., p. 81.

57. ' A. al-Malhūn̄ī, op.cit., p. 82.

58. 'A. al-Ğirārī, op.cit., p. 412 n.

59. ' A.al-Malhūnī, op.cit., p. 84. 
de l'islām et de l'imān ${ }^{60}$, thème très connu en théologie musulmane et qui a divisé les esprits depuis toujours ${ }^{61}$.

D'autre part, comme partout où la doctrine de la prédestination immuable et gratuite se fraie un chemin, planait ici aussi le spectre des «libertins», ibāhiyya, qui, tirant une des conséquences logiques de la doctrine du double décret immarcescible, n'ont cure des ceuvres, bonnes ou mauvaises. C'est contre un tel laxisme que s'étaient élevés aussi bien Ghazzāa $\overline{1}^{62}$ à Bagdad au $\mathrm{XI}^{\mathrm{e}}$ siècle que Calvin $^{63}$ à Genève au XVI ${ }^{\mathrm{e}}$ siècle. Et c'est ce à quoi Ghrabli feint à mi-mot d'accabler son contradicteur, en l'appelant à renoncer à l'hédonisme dans lequel le pousse $\operatorname{Satan}^{64}$. Mais en vérité, ni Tourkmani, ni Kaddour el-Alami, qui prit manifestement le parti de celui-ci, ne rejetaient les œuvres en tant que telles. Ils les considéraient seulement, malgré leur nécessité, comme inefficaces sans la foi qui leur donne leur sens, exactement comme le faisait l'islam primitif, à en juger par l'insistance du Coran sur le 'amal, et comme le fera plus tard le calvinisme aussi, qui cultivait l'idée, selon laquelle on devait «voir l'arbre à ses fruits». Et à l' origine de la foi, ils mettaient une décision divine éternelle. D'où leur adhésion sans retenue au dogme de la double prédestination comme un don gratuit de Dieu.

En fait ce que les deux poètes défendaient c'était aussi l'idée d'une immutabilité de la prédestination, ce qu'on appelle l'ithbāt en théologie musulmane, c'est-à-dire le fait que Dieu, comme dans le calvinisme examiné par Max Weber, ait parlé «une fois pour toutes». Sans jamais disparaître, cette idée née avec l'islam s'était néanmoins estompée à travers les siècles, au fur et à mesure que reculait la croyance en une stricte prédestination et que, surtout, $\mathrm{s}^{\text {'affirmait le providentialisme }}{ }^{65}$. Elle réapparaissait généralement

\section{Ibid., p. 82 .}

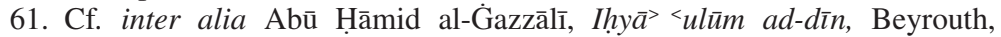
Dār al-kutub al-<ilmiyya, I, 1996, p. 138-148; L. Gardet, loc.cit., p. 61-123; David Künstlinger, «,Islām,,,,,Muslim,,,,,,aslama,, im Kurān», in Rocznik Orientalistyczny (1935), p. 128-137.

62. Otto Pretzl, Die Streitschrift des Ġazālī gegen die Ibāhīja, München, Bayerische Akademie der Wissenschaften, 1933.

63. Contre la secte phantastique et furieuse des libertins, Genève, Droz, 2005.

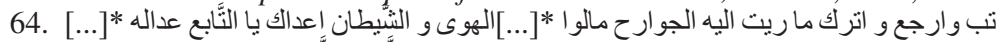

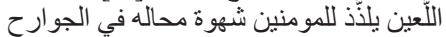

(A. al-Ğirārī, op.cit., p. 415). يسري سري الدم و يسكن في حشاها

65. Y. Djedi, «Prédestination...», loc.cit. 
quand ressurgissait le dogme du double décret, comme avec les Almohades, mais aussi lorsque celui-ci réclamait la recherche d'une certitudo salutis comme réponse logique au problème qu'elle pose. C'est ce qu' on retrouve dans le soufisme dans lequel baignent aussi les poètes «populaires» maghrébins. Ainsi cette profession de foi, au début du XVIII ${ }^{\mathrm{e}}$ siècle, du grand Mohammed Benmsayeb de Tlemcen: «L'élu ne saurait être réprouvé» ${ }^{66}$.

\section{LE PROBLÈME DE LA CERTITUDE : SALUT OU DÉPRAVATION?}

Il faut noter que la propension humaine au salut religieux exclut en principe presque toujours le sentiment d'une certitudo perditionis, bien que le doute quant au salut soit propre à toute expérience de quête sotériologique. En tout cas, l'édification religieuse ellemême, en islam comme ailleurs dans le protestantisme, pousse toujours vers le sentiment de conviction d'être sauvé, soit en insistant sur les œuvres, soit en déclarant le salut par la sola fides et par l'appartenance à la véritable «Église de Dieu», comme le font surtout le catholicisme et l'islam. C'est là justement l'un des angles d'attaque de Tourkmani: "Je dis que la foi est imperdable, fût-on adultère, voleur, brigand, homicide et buveur de $\operatorname{vin}^{67}$.» Tourkmani, on le voit, tire argument d'une des possibilités de l'islam orthodoxe le plus typiquement $«$ catholiques $»^{68}-$ pour parler comme Goldziher ${ }^{69}$.

Quant à la dépravation dans laquelle on voit avec «certitude» ses adversaires (généralement politiques), elle provient le plus souvent de cette conscience forgée par la «communauté de religiosité» qui soude ses membres par un sentiment de «sainteté» et, par conséquent, de «séparation» d'avec le monde. L'opposition entre la «poignée» d'élus et la massa perditionis que constitue «le reste de l'humanité» - ainsi l'avaient formulée les synodes protestants

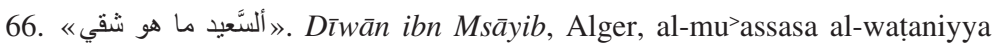
li-l-kitāb, 1989, p. 82.

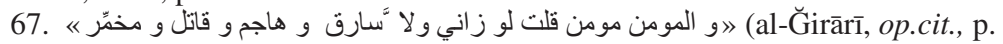
417).

68. Youcef Djedi, «Max Weber et Ignaz Goldziher: la "non rencontre"”, in Céline Trautmann-Waller (éd.), Ignác Goldziher. Un autre orientalisme?, Paris, Geuthner, 2011, p. 238.

69. «Katholische Tendenz und Partikularismus im Islam», loc.cit. 
de Westminster et de Dordrecht - ne demandera plus qu'à sortir au grand jour. Tels étaient, en effet, les «réprouvés » que recherchaient les épées des antiques musulmans, des soldats de Cromwell ou des cavaliers almohades.

Cette conscience de l'élection a donc pour corollaire l'existence d'êtres réprouvés de toute éternité. Et de cela aussi nos poètes «populaires» se font l'écho. Ainsi Abdelaziz el-Meghraoui dans sa qasīda «el-Mi ${ }^{\prime} \operatorname{radj}$ », d'ailleurs jalonnée de moments prédestinatianistes, dont celui-ci précisément : «Celui que Dieu a sauvé par sa grâce a eu la foi et a contemplé sa lumière [scil. le Prophète] * Et celui irrévocablement prédestiné à la réprobation aura l'enfer pour demeure ${ }^{70}$.» Dans une autre pièce - inédite «Chaytaniyya», d'un certain Boukhris (XVIII ${ }^{\mathrm{e}}$ s. $)^{71}$, c'est le «prince» des réprouvés lui-même, Satan, qui entre en scène pour confirmer l'ithbāt de sa propre damnation. Lorsque, dans cette étonnante fiction, le Prophète le presse, en effet, de «revenir vers Dieu, d'avoir honte * et de faire une génuflexion devant la tombe de notre père $A_{d a m^{72}}$ », Iblīs refuse catégoriquement, parce que «c'est pour l'enfer que le Seigneur des créatures, l'Immuable, m'a créé * Je ne me repens, ni reviens sur les actes de mon crime * Ainsi Dieu en a voulu et me l'a inéluctablement décrété * Il m'a créé pour le feu et a créé mon corps (à partir) de celui-ci ${ }^{73}$.»

Il est rare, en revanche, de rencontrer dans la réalité des cas d'une certitudo perditionis aussi établie. De ce point de vue, on peut s'étonner quelque peu de l'attitude du poète algérien Mohammed Baytar (1886-1919) ${ }^{74}$. Noceur invétéré qui a brûlé sa vie par tous les bouts, le voilà à l'article de la mort convaincu de sa propre damnation, pour ne pas s'être préparé, durant sa courte vie, à la «reddition des comptes ${ }^{75}$. Chantre des stupres et des beuveries, il n'était pas moins imbu de l'idée de prédestination et vivait déjà,

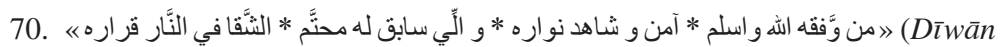
aš-šay $\underline{h}<A b d$ al-<Azīz al-Mag̉rāwī, op.cit., p. 108. Cf. également ibid., p. 123).

71. A. A. Dellai, op.cit., p. 230-231.

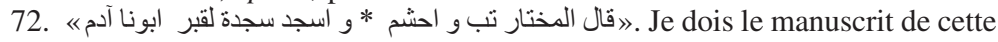
qașīda à l'obligeance de M. Abdelghani Mehdaoui.

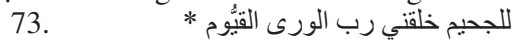

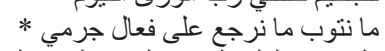

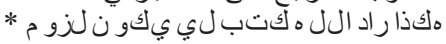

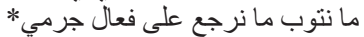

74. A. A. Dellai, op.cit., p. 89.

75. H. Boubakeur, op.cit., p. 290. 
pour ainsi dire, dans l'incertitude du salut; le sien propre d'abord, mais aussi celui de ses échansons et de ses maîtresses. À l'une de ces dernières, une jeune prostituée, dont il était pourtant en train de pleurer amèrement la mort, il adressa ces vers pour le moins très peu amènes: «Tu retrouveras tous les péchés que tu as commis * Tu en achèteras (alors) une place en enfer ${ }^{76}$.»

Qu'il y ait là une insinuation à la double prédestination, ne serait-ce qu'en raison de l'emploi de la «place» [mqam], terme par trop attaché aux traditions les plus fréquentes sur le double décret, ne paraît pas invraisemblable. Mais ce qui est intéressant aussi à noter dans le cas de Mohammed Baytar, c'est qu'il s'agisse évidemment d'un cas typique de la conception de l' «achat» de la damnation, de la sienne propre même, quand bien «il lui arrivait de se repentir, de redouter d'encourir la damnation qu'il méritait, lors du jugement dernier ${ }^{77}$.» Il est vrai par ailleurs qu'un tel «achat» est déjà établi par le Coran ${ }^{78}$ et que, de toute façon, il n'était pas inconnu à l'époque du jeune poète ${ }^{79}$.

Gardons-nous toutefois de prendre cette conception rigide de la prédestination pour monnaie courante. C'est plutôt le contraire qui prédomine. Ainsi, plus optimiste que Mohammed Baytar, Mohammed Benslimane ${ }^{80}$, par exemple, l'un des grands poètes du melhoun marocains du XVIII ${ }^{\mathrm{e}}$ siècle marocain, préférait-il se défaire du décret immuable. Sentant sa fin prochaine - il mourut à trentetrois ans - on le voit s'en remettre à la bonté d'Allah, qui pourrait aussi bien exaucer les prières des repentants et «solder» leurs comptes. Et si, comme Mohammed Baytar, il «regrette jusqu'aux larmes (s)es fautes et l'abandon de (s)es prières (quotidiennes)», c'est pour demander à Dieu d'avoir pitié de lui «le jour des tourments et du noir de la tombe» et de remplacer alors «(s)es péchés par une issue (bien)heureuse, husn $\bar{a}^{81}$.» Peut-être est-ce ici aussi une allusion à la prédestination déclarée par le Coran:

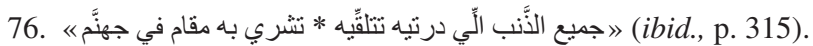

77. Ibid., p. 290.

78. Comparer e.g. avec les versets II, 16, 86, 90, 102, 175 ; III, 177 ; IV, 44, etc.

79. Mohamed Ben Cheneb, Proverbes arabes de l'Algérie et du Maghreb, Paris, E. Leroux, 1905-7, I, §§ 130, 452 et vraisemblablement aussi III, § 1990.

80. A. A. Dellai, op.cit., p. 225-227.

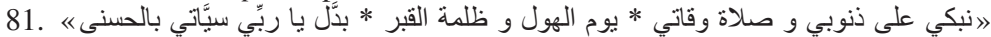
(A.A. Dellai, op.cit., p. 227). 
«Ceux-là à qui nous avons décrété la félicité [husnā] ils en seront certes éloignés [scil. de la géhenne] ${ }^{82}$.» On peut le supposer, en effet. En tout cas, les profondes convictions prédestinatianistes n'avaient pas empêché el-Meghraoui non plus, par crainte pour son sort futur, de supplier «le plus magnanime des magnanimes de ne pas dévoiler (le contenu) de mon livre (de comptes) * (Et) de remplacer ma grande gêne $\left[{ }^{<} u s r\right]$ par une issue aisée $[y u s r]^{83}$.»

Il a existé d'ailleurs aussi cette autre tendance qui consiste, non pas à rejeter la croyance en une double prédestination, mais à en entamer l'immutabilité, c'est-à-dire à en effacer l'ithbāt. Tendance certainement aussi ancienne que l'islam et qu'on fait remonter au moins jusqu'à Omar ibn al-Khațāâb. Ce grand șaḥābī, dont le nom cautionne quelques-unes des traditions prédestinatianistes les plus puissantes que renferment les grands recueils de hadiths, était, dit-on, connu aussi pour implorer Allah constamment de l'effacer de la liste des réprouvés, s'il y était, et de le compter parmi les bienheureux ${ }^{84}$. C'est le sens de la prière contenue dans ce chant, probablement d'origine andalouse, bien connu en Afrique du Nord: «Prends mon âme et inscris-la sur une tablette». Autrement dit, sur la bonne liste. Est-ce un hasard si c'est précisément le même Omar qui est à la tête de l'isnād d'un des plus célèbres hadiths sur la double prédestination gratuite? Un jour, non loin de Damas, à Djabiya, raconte-t-on, Omar fut apostrophé du haut de la chaire, alors qu'il prêchait justement sur la grâce d'Allah qui «guide» et «égare». Il faillit mettre à mort le dignitaire byzantin qui avait osé lui faire observer que Dieu ne saurait égarer personne inna Allāh lā yudill ahadāa, insinuant que la grâce est universelle. Il n'en fallut pas davantage pour irriter Omar qui lui répliqua: «C'est Dieu qui t'as créé et c'est Dieu qui t'a égaré, puis te fera mourir et te fera entrer en enfer (...).» Et d'ajouter : «Lorsqu'Allah [...] eut créé Adam, il éparpilla sa descendance et écrivit qui seront en paradis et

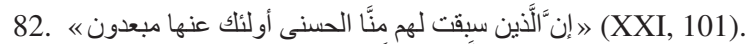

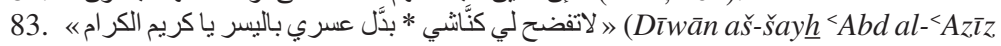
al-Magrāāw, op.cit., p. 139).

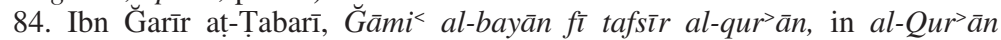
al-karīm (CD-ROM), Le Caire, Harf, 1998, XIII, 39, § 15532; <Abd Allāh b. Muslim ibn Qutayba, Ta`wīl muhtalaf al-hadīt , Beyrouth, Dār al-fikr, 1995, p. 19. Comparer avec la prière de David dans Psaumes, LXIX, 29. 
quelles seront leurs œuvres et qui seront en enfer et quelles seront leurs œuvres ${ }^{85}$.»

\section{En GUISE DE CONCLUSION}

Une recherche plus poussée dans le fonds inexploité de la poésie «populaire» nous montrerait encore mieux la place de la doctrine de la double prédestination dans la mentalité «populaire» jusqu'à l'orée de l'époque actuelle. Méconnue et ignorée, il n'est pas jusqu'à ses termini technici qui ne soient presque totalement oubliés. Aux $\mathrm{XVI}^{\mathrm{e}}$ et $\mathrm{XVII}{ }^{\mathrm{e}}$ siècles, les morisques qui étaient en contact avec les huguenots étaient conscients de leur communauté de vue sur ce point de doctrine avec le protestantisme. Celui-ci était traversé alors par de larges débats sur le decretum horribile. Mais le fait est que cette doctrine était profondément ancrée dans l'islam maghrébin, qui sur ce point précisément a été indiscutablement marqué par la théo-idéologie almohade. La littérature arabe «savante» n'apporte plus d'éclairage, ou si peu, sur cette croyance qui, très souvent, a accompagné de grands bouleversements sociaux et politiques, en Europe comme en Afrique du Nord et au Moyen-Orient (le grand Ghazzālī en avait bien souligné le danger dans sa diatribe contre les Bātiniyya). On en sent encore des relents sous le règne du sultan marocain Ahmed el-Mansour Eddahabi (1578-1603), par exemple, ce qui indique que la dynastie des Saadides (15541660) n'était pas complètement détachée de la culture politique et religieuse almohade. On peut le voir, par exemple, à travers la rhétorique maniée par le renégat Mahmoud Zarqoun ${ }^{86}$, parti à la tête de l'expédition envoyée par Ahmed el-Mansour Eddahabi à la conquête du royaume de Gao et de Tombouctou ${ }^{87}$. N'est-ce pas intéressant de rappeler que Abdelaziz el-Meghraoui, par

85. Abū Bakr Muhammad ibn al-Husayn al-Ağurrī, aš- ̌̌arī<a, s.l., Matba<at as-sunna-l- muḥammadiyya, 1950, p. 200-201; Abū Bakr ibn al-< Arab̄i, Aḥkām al-Qur>ān (éd. <A.M. al- Biğāāī), Beyrouth, Dār al- Ğ̄ill, s.d., II, p. 800.

86. Évariste Lévi-Provençal, «Un document inédit sur l'expédition sa<dide au Soudan », in Arabica (1955), II, p. 89-110.

87. W.M.G. de Slane, «Conquête du Soudan par les Marocains en l'an 999 (1500-1 de J.C.). Récit extrait de l'ouvrage d'un historien arabe», in Revue Africaine (1856/57), $\mathrm{n}^{\circ} 1$, p. 287-298. 
exemple, est l'auteur de thrènes ${ }^{88}$ sur «le bienheureux d'entre tous, $a l-a s^{<} a d^{89} »$, le sultan el-Mansour Eddahabi? Mais plus intéressant encore pour ce qui nous concerne est cette «bête», annoncée, pour la fin des temps, par le Coran ${ }^{90}$, et qui, d'après les traditions dont s'était inspiré le grand poète marocain, «sortira parmi les gens, (pour les) marquer et faire le départ entre l'élu et le damné $»^{91}$. On ne peut s'empêcher de penser alors aux journées du terrible «tri», mayz, effectué par le «Mahdī de la fin des temps », Mohammed ibn Toumert, et ses «saints».

youcef.djedi@uvsq.fr

88. Dīwān $a \check{s}-\check{s} a y \underline{h}<$ Abd al-<Azīz al-Mag்rāwī, op.cit., p. 303-312.

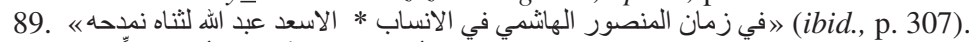

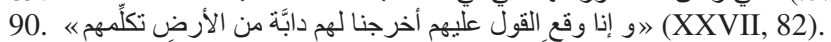

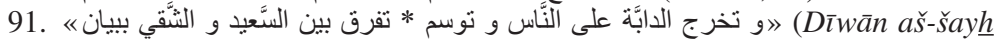
<Abd al-<Azīz al-Mag̀r rāwī, op.cit., p. 143). 